\title{
Comment to the paper by L. May: Lack of uniformity in the clinical assessment of children with lipomyelomeningocele. A review of the literature and recommendations for the future
}

\author{
Concezio Di Rocco
}

Received: 22 February 2013 / Accepted: 25 February 2013 / Published online: 20 March 2013

(C) Springer-Verlag Berlin Heidelberg 2013

This paper by Lindy May and co-workers is a timely review of the limits currently experienced by all of us in evaluating the outcome of patients with "lipomyelomeningocele". It is based on a meta-analysis of the literature of the last three decades carried out to research and analyze the reliability of the commonly utilized assessment tools. As expected, the series reviewed, all retrospective in nature, are too heterogeneous to be compared. Even more confusing is the inconsistencies of the definition "lipomyelomeningocele" loosely applied to a variety of malformations which differ in terms of pathogenesis, severity, and surgical difficulties. The "suggested tables" reflect the rather pessimistic conclusion that the paper has generated, that is the necessity of focusing on rather simple evaluations: yes/not, stablel improved/worsened rather than attempting to use more sophisticated and difficult to apply scales.

In my opinion, it is timely that the pediatric neurosurgical community faces the problem of the surgical indication in this spectrum of spinal malformations taking into account the natural history and the surgical outcomes. First of all, however, we should agree on the diagnosis and the way of labelling these malformations as the plethora of definitions currently existing, generates an obvious problem. In such a regard, the classification proposed by Tortori-Donati may at least subdivide the affected subjects into two main groups, those with an intact dura and those with a defective dura mater, which differ embryologically and would be easier to analyze and compare.

We should also discuss which examinations should be done and at which age in the attempt to focus on reliable and comparable results by generating subgroups, e.g., newborns, toddlers, adolescents and adults, the comparison of which could be more trustworthy.

As the malformations, here considered, are relatively uncommon, it is clear that only a multicentric study could answer the numerous still open questions. In other terms, only a prospective study based on generally accepted criteria of evaluation at diagnosis and, successively, on shared assessment tools during the follow-up observation might provide the right answers. Hopefully, the Education Committees of the various Societies which recognize Child's Nervous System as their official journal could accept to be involved in such a task

Concezio Di Rocco

cdirocco@rm.unicatt.it

C. Di Rocco $(\bowtie)$

Paediatric Neurosurgery, Catholic University Medical School,

A. Gemelli Hospital, L. go A. Gemelli 8,

00168 Rome, Italy

e-mail: cdirocco@rm.unicatt.it 